\title{
Sintomas ansiosos e depressivos e sua correlação com intensidade da dor em pacientes com neuropatia periférica
}

\author{
Anxious and depressive symptoms and their correlation with pain severity \\ in patients with peripheral neuropathy
}

\author{
Israel Soares Pompeu de Sousa Brasil ${ }^{1}$, Milena Pereira Pondé ${ }^{2}$
}

${ }^{1}$ Acadêmico de Medicina. Escola Bahiana de Medicina e Saúde Pública, Salvador, BA. ${ }^{2}$ Professora adjunta, Escola Bahiana de Medicina e Saúde Pública.

\section{Resumo}

Introdução: As neuropatias periféricas incluem disfunções sensitivo-motoras e quadros de dor crônica que podem propiciar a eclosão de transtornos psiquiátricos, como a ansiedade e a depressão. Este estudo visa estimar a frequência de sintomas ansiosos e depressivos entre pacientes com neuropatia periférica, correlacionando-os com a intensidade da dor.

Método: Estudo de corte transversal realizado em ambulatório docente assistencial de neurologia entre abril de 2006 e março de 2007. Foram utilizados como instrumentos de avaliação a Escala Hospitalar de Ansiedade e Depressão e a Escala Analógica Visual de Dor para avaliação da dor. A amostra foi composta por 54 pacientes.

Resultados: Constatou-se uma frequência de 68,5\% $(n=37)$ de sintomas ansiosos e 51,9\% $(n=28)$ de sintomas depressivos. Dor intensa foi relatada por $57,4 \%$ dos pacientes. Houve correlação positiva entre a intensidade da dor e a de sintomas ansiosos e depressivos (p $\leq 0,05)$. Presença de cefaleia, trauma e história familiar de doença psiquiátrica também estava associada positivamente aos sintomas ansiosos e depressivos.

Conclusões: Os resultados demonstram uma elevada frequência de sintomas ansiosos e depressivos em pacientes com neuropatia periférica, havendo uma correlação positiva com a gravidade da dor.

Descritores: Ansiedade, depressão, dor, medição da dor, doenças do sistema nervoso periférico.

\begin{abstract}
Introduction: Peripheral neuropathies include sensory-motor dysfunctions and chronic pain that may trigger psychiatric disorders such as anxiety and depression. The objective of the present study was to estimate the frequency of anxious and depressive symptoms among patients with peripheral neuropathies, correlating them with pain severity.

Methods: Cross-sectional study conducted in an outpatient neurology clinic between April 2006 and March 2007. The Hospital Anxiety and Depression Scale and the Pain Visual Analogue Scale were used to evaluate pain. The sample included 54 patients.

Results: We found a frequency of $68.5 \%(\mathrm{n}=37)$ of anxious symptoms and $51.9 \%(\mathrm{n}=28)$ of depressive symptoms. Severe pain was reported by $57.4 \%$ of the patients. There was a positive correlation between pain severity and anxious and depressive symptoms $(\mathrm{p} \leq 0.05)$. Headache, trauma and family history of psychiatric disease were also associated with anxious and depressive symptoms.

Conclusions: Our findings demonstrate a high frequency of anxious and depressive symptoms in patients with peripheral neuropathies. Such symptoms are positively correlated with pain severity.
\end{abstract}

Keywords: Anxiety, depression, pain, pain measurement, peripheral nervous system diseases.

\section{Correspondência:}

Israel Soares Pompeu de Sousa Brasil, Rua Tenente Fernando Tuy, 269/1001, Itaigara, CEP 41810-780, Salvador, BA. Tel.: (71) 3358.0949. E-mail: israelpompeu@, yahoo.com.br

Bolsa de Iniciação Científica da Fundação de Amparo à Pesquisa do Estado da Bahia (FAPESB) - protocolo nº $0452 / 2006$.

Não há conflitos de interesse associados à publicação deste artigo.

Copyright (C) Revista de Psiquiatria do Rio Grande do Sul - APRS 


\section{Introdução}

A associação entre transtornos mentais e doenças físicas se deve tanto a fatores psicossociais quanto biológicos. Dentre os primeiros, ressaltam-se a frustração na realização de desejos e necessidades, agravamento de conflitos intrapsíquicos, inadequação dos mecanismos de defesa, perda do sentimento de autoestima, alteração da imagem corporal e isolamento social ${ }^{1}$. A ruptura do ciclo sono-vigília e o uso de medicamentos e de procedimentos que afetam o sistema nervoso central figuram entre os fatores biológicos ${ }^{1}$. $\mathrm{O}$ transtorno mental pode estar associado a problemas físicos, assim como alterações orgânicas podem resultar em disfunções psíquicas. A depressão e a ansiedade fóbica, por exemplo, podem estar associadas a um maior risco de infarto do miocárdio ${ }^{2}$. Além disso, reações de ajustamento são comuns em pacientes internados ${ }^{3}$, e depressão e labilidade emocional ocorrem com frequência em pessoas com afecções vasculares cerebrais ${ }^{4}$.

Dois transtornos psiquiátricos se destacam ao se abordar a relação estabelecida entre os fatores orgânicos e psíquicos. A ansiedade, que frequentemente se associa a situações novas e desconhecidas, como é o caso do adoecimento, tem prevalência elevada em serviços de atenção primária: $13,8 \%$ na Espanha; 19,2\% no Canadá e 21,8\% nos EUA ${ }^{5}$. A depressão pode ser confundida com a doença orgânica de base, uma vez que o sofrimento e a incapacidade funcional favorecem o surgimento de sintomas como insônia, fadiga e perda da libido, mesmo quando não se estabelece o diagnóstico de depressão ${ }^{6}$. Os sintomas mais comuns da depressão em pacientes com doenças físicas são perda do interesse nas pessoas, pessimismo, indecisão, irritabilidade, anedonia, insônia, baixa autoestima e desesperança ${ }^{7-9}$.

$\mathrm{O}$ acometimento dos nervos periféricos se insere nesse contexto por envolver uma ampla variedade de sintomas, incluindo dor crônica e disfunções sensitivo-motoras. Em estudo com pacientes portadores de neuropatia diabética, foi observado que 60,4\% apresentava ansiedade e 50,6\% depressão, sendo os sintomas proporcionais à intensidade da dor sofrida ${ }^{10}$. Sintomas depressivos, medidos pela Escala Hospitalar de Ansiedade e Depressão, puderam ser associados à neuropatia ${ }^{11}$. A dor neuropática, presente em grande parte das neuropatias periféricas, pode ser fator causador ou agravante de sintomas psiquiátricos. A presença de dor aguda também pode desencadear uma reação ansiosa, levando a rebaixamento do limiar de dor ${ }^{12}$. A comorbidade de transtornos psiquiátricos com queixas de dor crônica é alta. Algum tipo de transtorno de humor foi identificado em $21,7 \%$ de uma amostra com pacientes apresentando dor crônica ${ }^{13}$. As prevalências de dor em coortes de depressão e de depressão em coortes de dor são mais altas do que naquelas em que tais condições são avaliadas isoladamente ${ }^{14}$. Sítios múltiplos de dor também se mostraram associados a transtornos de humor e de ansieda$\mathrm{de}^{15}$. Outro fator de risco são as comorbidades como diabetes, doença cardíaca e câncer, que demonstraram ter um papel importante na incapacidade proporcionada pelas condições dolorosas crônicas ${ }^{16}$, potencializando, assim, a sintomatologia depressiva e ansiosa. Não há definições na literatura quanto a qual transtorno insere maior risco, apesar de os transtornos de ansiedade parecerem estar mais fortemente associados a queixas de dor do que os transtornos de humor ${ }^{17}$.

Com base nessas informações, este estudo busca identificar a frequência de sintomas ansiosos e depressivos em pacientes de baixa renda portadores de neuropatia periférica, além de correlacionar a intensidade da dor sentida pelo paciente com a intensidade dos sintomas psiquiátricos.

\section{Método}

Foi feito um estudo de corte transversal, realizado entre abril de 2006 e março de 2007 em pacientes com neuropatia periférica atendidos no ambulatório de neuropatias periféricas da Fundação de Neurologia e Neurocirurgia Instituto do Cérebro (Salvador, BA). Trata-se de um serviço docente assistencial que atende gratuitamente pessoas de baixa renda. Foram incluídos todos os pacientes que compareceram ao serviço durante o período da coleta de dados, com idade $\geq$ a 18 anos, que assinaram o consentimento informado e que apresentavam funções cognitivas preservadas para responder ao questionário. Foram excluídos os pacientes que não apresentavam quadro suspeito de neuropatia periférica, menores de 18 anos e que se recusassem a participar da pesquisa.

Os instrumentos de avaliação utilizados foram: ficha para coleta de dados sociodemográficos, informações sobre a doença de base e antecedentes médicos, Escala Hospitalar de Ansiedade e Depressão e Escala Analógica Visual de Dor. A Escala Hospitalar de Ansiedade e Depressão foi escolhida por ser um instrumento que não envolve sintomas somáticos, que poderiam confundir o diagnóstico de transtorno de humor ou de ansiedade ${ }^{18}$. A versão em português foi validada para pacientes ambulatoriais, resultando em sensibilidade de $74,2 \%$ e especificidade de $74 \%$ para a subescala de ansiedade e sensibilidade de $85,7 \%$ e especificidade de $72,4 \%$ para a subescala de depressão ${ }^{19}$. O ponto de corte para ambas as escalas foi 8 , sendo o mesmo utilizado no presente trabalho. Os questionários foram aplicados oralmente para toda a amostra, em função de terem sido incluídos pacientes não-alfabetizados. Para avaliar a intensidade da dor foi aplicada a Escala Analógica Visual de Dor, sendo 0 (zero) indicador de ausência de dor e 10 de dor insuportável. Trata-se de um método simples e de fácil aferição da intensidade da dor, compatível com o grau de instrução da população abordada. Classificou-se a pontuação em três categorias, como foi feito em estudo de Tölle et al. ${ }^{20}$ : de 1 a 3 , dor leve; de 4 a 7 , dor moderada; e de 8 a 10, dor intensa. $\mathrm{O}$ paciente foi abordado pelo investigador, um acadêmico de medicina treinado para tal.

O estudo foi aprovado pelo Comitê de Ética em Pesquisa da Fundação Bahiana para Desenvolvimento das Ciências, através do protocolo $\mathrm{n}^{\circ} 25 / 2006$, e teve o financiamento provido pela Fundação de Amparo à Pesquisa do Estado da Bahia (FAPESB). Todos os pacientes incluídos receberam e assinaram o termo de consentimento livre e esclarecido. 
Para a análise dos dados, foi utilizado o software Statistical Package for the Social Sciences (SPSS) versão 12.0. Para análise descritiva, foram utilizados os parâmetros da estatística descritiva, adotando-se as medidas usuais de tendência central (média, mediana e moda) e de dispersão (amplitude, variância, desvio padrão e coeficiente de variação) e cálculos de frequências simples e relativas. Para as variáveis contínuas com distribuição normal, foi utilizado o teste $t$ de Student; para as frequências das variáveis categóricas, o teste do qui-quadrado e teste exato de Fisher. Análise por regressão linear foi feita para correlacionar a intensidade dos sintomas de ansiedade e depressão com a intensidade da dor referida. O nível de significância adotado foi de 5\%.

\section{Resultados}

Foram abordados, para a composição da amostra, 68 pacientes, sendo que 54 atenderam aos critérios de inclusão. Dentre os 14 pacientes excluídos, dois eram menores de 18 anos, sete não portavam doenças classificadas como neuropatia periférica (três com miopatia, três com mielopatia e um com neuralgia do trigêmeo de possível causa central), um tinha doença de base topográfica ainda a esclarecer, um tinha doença de Alzheimer em estágio avançado e três se recusaram a participar do estudo.

A Tabela 1 resume os dados sociodemográficos e clínicos. A idade média do grupo foi de $44,5 \pm 10,4$ anos, sendo que $74 \%$ tinham até 50 anos de idade. A maior parte (90,7\%) tinha renda mensal de, no máximo, três salários mínimos (salário mínimo de $\mathrm{R} \$ 350,00$ à época da coleta), sendo que a maioria $(85,2 \%)$ não exercia o emprego (desempregados, dispensados ou aposentados). Em relação aos dados clínicos, as neuropatias periféricas mais frequentemente observadas foram as radiculopatias $(41,5 \%)$ e a síndrome do túnel do carpo $(30,2 \%)$. A maior parte da amostra foi composta de doentes crônicos: a duração média de doença foi de $5,2 \pm 4,3$ anos, sendo que $38,9 \%$ eram portadores da enfermidade há mais de 5 anos. A presença de comorbidades foi observada em $83,3 \%$ dos pacientes: cefaleia foi referida por $29,6 \%$ da amostra; hipertensão arterial sistêmica (HAS), por 24\%; e 22,2\% afirmaram ser tabagistas. Acompanhamento psiquiátrico era feito por $20,4 \%$ dos pacientes, dos quais $63,6 \%$ referiram depressão como razão para o acompanhamento. $\mathrm{O}$ uso de antidepressivos tricíclicos (amitriptilina ou nortriptilina) era feito por $68,5 \%$ dos pacientes; $19 \%$ usavam anti-inflamatórios não-esteroides; $17,1 \%$ complexos vitamínicos; $13,3 \%$ relaxantes musculares e 13,3\% anticonvulsivantes. A polifarmácia foi observada em $70,3 \%$ dos pacientes, sendo o número médio de medicamentos utilizados pelos pacientes de $2,4 \pm 1,2$.

A pontuação $\geq 8$ pela subescala de ansiedade da Escala Hospitalar de Ansiedade e Depressão (HAD-A) foi atingida por $68,5 \%$ da amostra, com pontuação média de $9,4 \pm 4,6$. Os sintomas ansiosos foram mais comuns entre os pacientes que se encontravam na faixa etária de 31 a 50 anos; os que portavam
Tabela 1 - Distribuição da amostra em relação aos principais dados sociodemográficos e clínicos

\begin{tabular}{|c|c|}
\hline Característica & n (\%) \\
\hline \multicolumn{2}{|l|}{ Sexo } \\
\hline Masculino & $26(48,1)$ \\
\hline Feminino & $28(51,9)$ \\
\hline \multicolumn{2}{|l|}{ Faixa etária } \\
\hline 18 a 30 anos & $3(5)$ \\
\hline 31 a 50 anos & $37(69)$ \\
\hline Mais de 50 anos & $14(26)$ \\
\hline \multicolumn{2}{|l|}{ Estado civil } \\
\hline Solteiro(a) & $17(31,4)$ \\
\hline Casado(a) & $29(53,7)$ \\
\hline Separado(a)/viúvo(a) & $8(14,9)$ \\
\hline \multicolumn{2}{|l|}{ Filhos } \\
\hline Sim & $47(87)$ \\
\hline Não & $7(13)$ \\
\hline \multicolumn{2}{|l|}{ Grau de escolaridade } \\
\hline Até $1^{\circ}$ grau completo & $30(55,6)$ \\
\hline Acima de $1^{\circ}$ grau & $24(44,4)$ \\
\hline \multicolumn{2}{|l|}{ Procedência } \\
\hline Capital (Salvador) & $44(81,5)$ \\
\hline Interior (BA) & $10(18,5)$ \\
\hline \multicolumn{2}{|l|}{ Estado empregatício } \\
\hline Empregado & $8(14,8)$ \\
\hline Desempregado & $8(14,8)$ \\
\hline Dispensado & $9(16,6)$ \\
\hline Aposentado & $29(53,8)$ \\
\hline \multicolumn{2}{|l|}{ Renda mensal familiar } \\
\hline Até 1 salário mínimo & $10(18,5)$ \\
\hline 1 a 3 salários mínimos & $39(72,2)$ \\
\hline Mais que 3 salários mínimos & $5(9,3)$ \\
\hline \multicolumn{2}{|l|}{ Faixa de tempo de doença } \\
\hline$<1$ ano & $4(7,4)$ \\
\hline Entre 1 e 3 anos & $16(29,6)$ \\
\hline$>3$ anos & $34(63)$ \\
\hline \multicolumn{2}{|l|}{ Lesão decorrente de trauma } \\
\hline Sim & $8(14,8)$ \\
\hline Não & $46(85,2)$ \\
\hline \multicolumn{2}{|l|}{ Comorbidades } \\
\hline Sim & $45(83,3)$ \\
\hline Não & $9(16,7)$ \\
\hline \multicolumn{2}{|l|}{ Acompanhamento com psiquiatra } \\
\hline Sim & $11(20,4)$ \\
\hline Não & $43(79,6)$ \\
\hline \multicolumn{2}{|c|}{ História familiar de doença psiquiátrica } \\
\hline $\operatorname{Sim}$ & $22(44)$ \\
\hline Não & $26(52)$ \\
\hline Não sabe informar & $6(4)$ \\
\hline
\end{tabular}


lesões provenientes de trauma; os que referiam cefaleia; os que tinham antecedentes familiares de doença psiquiátrica; os que sofriam de dor intensa; e os que apresentavam sintomas depressivos ( $\mathrm{p} \leq 0,05)$ (Tabela 2). Já 51,9\% dos pacientes tiveram escores $\geq 8$ pela subescala de depressão (HAD-D), tendo uma pontuação média de $8,2 \pm 4,3$. Os sintomas de depressão

Tabela 2 - Distribuição da amostra segundo a pontuação da Escala Hospitalar de Ansiedade e Depressão quanto aos sintomas ansiosos e depressivos entre as principais variáveis socioeconômicas e clínicas abordadas

\begin{tabular}{|c|c|c|c|c|c|c|}
\hline Característica & 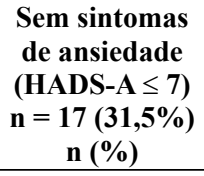 & 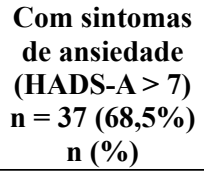 & $\mathbf{p}$ & $\begin{array}{c}\text { Sem sintomas } \\
\text { depressivos } \\
(\text { HADS-D } \leq 7) \\
\mathrm{n}=\mathbf{2 6}(\mathbf{4 8 , 1 \% )} \\
\text { n }(\%)\end{array}$ & $\begin{array}{c}\text { Com sintomas } \\
\text { depressivos } \\
(\text { HADS-D }>7) \\
\text { n= 28 }(51,9 \%) \\
\text { n }(\%) \\
\end{array}$ & $\mathbf{p}$ \\
\hline \multicolumn{7}{|l|}{ Sexo } \\
\hline Masculino & $8(47,2)$ & $18(48,6)$ & \multirow[t]{2}{*}{0,574} & $15(57,6)$ & $11(39,2)$ & \multirow[t]{2}{*}{0,140} \\
\hline Feminino & $9(52,8)$ & $19(51,4)$ & & $11(42,4)$ & $17(60,8)$ & \\
\hline \multicolumn{7}{|l|}{ Faixa etária } \\
\hline $18-30$ & $3(17,6)$ & $0(0)$ & \multirow[t]{3}{*}{0,011} & $3(11,5)$ & $0(0)$ & \multirow[t]{3}{*}{0,012} \\
\hline $31-50^{*}$ & $8(47,2)$ & $29(78,4)$ & & $13(50)$ & $24(85,7)$ & \\
\hline$>50$ & $6(35,2)$ & $8(21,6)$ & & $10(38,5)$ & $4(14,3)$ & \\
\hline \multicolumn{7}{|l|}{ Estado civil } \\
\hline Solteiro(a) & $5(29,4)$ & $12(32,4)$ & \multirow[t]{3}{*}{0,470} & $9(34,7)$ & $8(28,5)$ & \multirow[t]{3}{*}{0,771} \\
\hline Casado(a) & $8(47,2)$ & $21(56,8)$ & & $14(53,8)$ & $15(53,5)$ & \\
\hline Separado(a)/viúvo(a) & $4(23,4)$ & $4(10,8)$ & & $3(11,5)$ & $5(18)$ & \\
\hline \multicolumn{7}{|l|}{ Grau de escolaridade } \\
\hline Até $1^{\circ}$ grau completo & $7(41)$ & $23(62)$ & \multirow[t]{2}{*}{0,126} & $15(57,6)$ & $15(53,5)$ & \multirow[t]{2}{*}{0,488} \\
\hline Acima de $1^{\circ}$ grau & $10(59)$ & $14(38)$ & & $11(42,4)$ & $13(46,5)$ & \\
\hline \multicolumn{7}{|l|}{ Renda mensal familiar } \\
\hline Menos que 1 salário mínimo & $2(11,7)$ & $8(21,6)$ & \multirow[t]{3}{*}{0,657} & $2(7,6)$ & $8(28,5)$ & \multirow[t]{3}{*}{0,138} \\
\hline 1 a 3 salários mínimos & $13(76,6)$ & $26(70,4)$ & & $21(80,7)$ & $18(64,5)$ & \\
\hline Mais que 3 salários mínimos & $2(11,7)$ & $3(8)$ & & $3(11,5)$ & $2(7)$ & \\
\hline \multicolumn{7}{|l|}{ Faixa de tempo de doença } \\
\hline$<1$ ano & $1(5,8)$ & $3(8,3)$ & \multirow[t]{3}{*}{0,956} & $1(3,8)$ & $3(10,7)$ & \multirow[t]{3}{*}{0,629} \\
\hline Entre $1-3$ anos & $5(29,4)$ & $11(29,7)$ & & $8(30,7)$ & $8(28,5)$ & \\
\hline$>3$ anos & $11(64,8)$ & $23(62)$ & & $17(65,5)$ & $17(60,8)$ & \\
\hline \multicolumn{7}{|l|}{ Lesão decorrente de trauma } \\
\hline Sim & $0(0)$ & $8(21,6)$ & 0,037 & $1(3,8)$ & $7(25)$ & 0,033 \\
\hline \multicolumn{7}{|l|}{ Cefaleia } \\
\hline Sim & $2(11,7)$ & $14(38)$ & 0,048 & $4(15,3)$ & $12(42,8)$ & 0,027 \\
\hline \multicolumn{7}{|l|}{ Hipertensão arterial sistêmica } \\
\hline $\operatorname{Sim}$ & $3(17,6)$ & $10(27)$ & 0,350 & $6(23)$ & $7(25)$ & 0,561 \\
\hline \multicolumn{7}{|l|}{ Tabagismo } \\
\hline Sim & $4(23,4)$ & $8(21,6)$ & 0,567 & $6(23)$ & $6(21,5)$ & 0,571 \\
\hline \multicolumn{7}{|l|}{ Alcoolismo } \\
\hline Sim & $1(5,8)$ & $1(2,7)$ & 0,535 & $2(7,6)$ & $0(0)$ & 0,227 \\
\hline \multicolumn{7}{|l|}{ Acompanhamento com psiquiatra } \\
\hline Sim & $1(5,8)$ & $10(27)$ & 0,071 & $4(15,3)$ & $7(25)$ & 0,297 \\
\hline História familiar de doença psiq & & & & & & \\
\hline Sim & $3(18,8)$ & $19(59,3)$ & 0,016 & $8(32)$ & $14(60,8)$ & 0,047 \\
\hline Intensidade da dor & & & & & & \\
\hline Ausente/leve & $5(29,4)$ & $3(8,3)$ & 0,045 & $7(26,9)$ & $1(3,5)$ & 0,003 \\
\hline Moderada & $6(35,3)$ & $9(24,3)$ & & $10(38,5)$ & $5(18)$ & \\
\hline Intensa* & $6(35,3)$ & $25(67,4)$ & & $9(34,6)$ & $22(78,5)$ & \\
\hline $\begin{array}{l}\text { Associação com sintomas } \\
\text { depressivos/ansiosos }\end{array}$ & & & & & & \\
\hline Sim & $3(17,6)$ & $25(67,4)$ & 0,001 & $12(46,2)$ & $25(89,2)$ & 0,001 \\
\hline
\end{tabular}

HADS = Escala Hospitalar de Ansiedade e Depressão.

* Grupo de pacientes para o qual o valor de p se refere entre as variáveis com três categorias.

† Os pacientes que não souberam informar sobre antecedentes familiares de doença psiquiátrica $(\mathrm{n}=6)$ não foram considerados na análise. 
Tabela 3 - Distribuição da amostra quanto à Escala Analógica Visual de Dor entre as principais variáveis socioeconômicas e clínicas abordadas no estudo

\begin{tabular}{|c|c|c|c|}
\hline Característica & $\begin{array}{c}\text { Dor ausente/leve/moderada } \\
\text { (EAVD 0-6) } \\
\mathbf{n}=23(42,6 \%) \\
\text { n (\%) } \\
\end{array}$ & 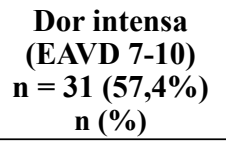 & $\mathbf{p}$ \\
\hline \multicolumn{4}{|l|}{ Sexo } \\
\hline Masculino & $14(60,8)$ & $12(38,7)$ & 0,091 \\
\hline Feminino & $9(39,2)$ & $19(61,3)$ & \\
\hline \multicolumn{4}{|l|}{ Faixa etária } \\
\hline $18-30$ & $3(13)$ & $0(0)$ & 0,074 \\
\hline $31-50$ & $13(56,5)$ & $24(77,4)$ & \\
\hline$>50$ & $7(30,5)$ & $7(22,6)$ & \\
\hline \multicolumn{4}{|l|}{ Estado civil } \\
\hline Solteiro(a) & $9(39,2)$ & $8(25,8)$ & 0,412 \\
\hline Casado(a) & $12(52)$ & $17(54,8)$ & \\
\hline Separado(a)/viúvo(a) & $2(8,8)$ & $6(19,4)$ & \\
\hline \multicolumn{4}{|l|}{ Grau de escolaridade } \\
\hline Até $1^{\circ}$ grau completo & $13(56,5)$ & $17(54,8)$ & 0,562 \\
\hline Acima de $1^{\circ}$ grau & $10(43,5)$ & $14(45,2)$ & \\
\hline \multicolumn{4}{|l|}{ Renda mensal familiar } \\
\hline Menos que 1 salário mínimo & $2(8,8)$ & $8(25,8)$ & 0,259 \\
\hline 1 a 3 salários mínimos & $19(82,4)$ & $20(64,5)$ & \\
\hline Mais que 3 salários mínimos & $2(8,8)$ & $3(9,7)$ & \\
\hline \multicolumn{4}{|l|}{ Faixa de tempo de doença } \\
\hline$<1$ ano & $1(4,5)$ & $3(9,7)$ & 0,640 \\
\hline Entre 1 e 3 anos & $8(34,7)$ & $8(25,8)$ & \\
\hline$>3$ anos & $14(60,8)$ & $20(64,5)$ & \\
\hline \multicolumn{4}{|l|}{ Lesão decorrente de trauma } \\
\hline Sim & $3(13)$ & $5(16)$ & 0,524 \\
\hline \multicolumn{4}{|l|}{ Cefaleia } \\
\hline $\operatorname{Sim}$ & $4(17,6)$ & $12(38,7)$ & 0,080 \\
\hline \multicolumn{4}{|l|}{ Hipertensão arterial sistêmica } \\
\hline Sim & $7(30,5)$ & $6(19,4)$ & 0,267 \\
\hline \multicolumn{4}{|l|}{ Tabagismo } \\
\hline Sim & $7(30,5)$ & $5(16)$ & 0,179 \\
\hline \multicolumn{4}{|l|}{ Alcoolismo } \\
\hline Sim & $2(8,8)$ & $0(0)$ & 0,177 \\
\hline \multicolumn{4}{|l|}{ Acompanhamento com psiquiatra } \\
\hline Sim & $4(17,6)$ & $7(22,6)$ & 0,454 \\
\hline \multicolumn{4}{|c|}{ História familiar de doença psiquiátrica* } \\
\hline Sim & $9(42,8)$ & $13(48)$ & 0,440 \\
\hline \multicolumn{4}{|l|}{ Sintomas de ansiedade } \\
\hline Sim & $12(52,2)$ & $25(80,6)$ & 0,027 \\
\hline \multicolumn{4}{|l|}{ Sintomas de depressão } \\
\hline $\operatorname{Sim}$ & $6(26)$ & $22(70,9)$ & 0,001 \\
\hline \multicolumn{4}{|c|}{ Sintomas de ansiedade + sintomas depressivos } \\
\hline $\operatorname{Sim}^{\dagger}$ & $5(21,7)$ & $20(64,5)$ & 0,006 \\
\hline Não & $7(30,5)$ & $6(19,5)$ & \\
\hline Sem sintomas & $11(47,8)$ & $5(16)$ & \\
\hline
\end{tabular}

EAVD $=$ Escala Analógica Visual de Dor.

* Os pacientes que não souberam informar sobre antecedentes familiares de doença psiquiátrica $(\mathrm{n}=6)$ não foram considerados na análise.

† Grupo de pacientes para o qual o valor de p se refere nas variáveis com três categorias. 
foram mais frequentes entre os mesmos grupos de pacientes associados aos sintomas de ansiedade (31-50 anos; trauma; cefaleia; antecedentes familiares de doença psiquiátrica; dor intensa), além daqueles que atingiram pontuação para sintomas ansiosos ( $\mathrm{p} \leq 0,05)$ (Tabela 2).

Quase metade dos pacientes (46,3\%) apresentava concomitantemente sintomas de ansiedade e depressão. A pontuação média de sintomas ansiosos entre esses pacientes foi de 13,1 \pm 3 , sendo significativamente maior quando comparada com a dos pacientes com sintomas ansiosos isoladamente $(\mathrm{p}<0,001)$. A pontuação média de sintomas depressivos foi de 11,38 $\pm 2,2$, sem diferença significativa em relação à pontuação dos pacientes apenas com sintomas de depressão.

Com relação à intensidade da dor medida pela Escala Analógica Visual de Dor, 5,6\% da amostra relatavam ausência de dor; 9,3\% dor leve (1 a 3 na Escala Analógica Visual de Dor); 27,8\% dor moderada (4 a 6 na Escala Analógica Visual de Dor) e 57,4\% relatavam dor intensa (7 a 10). A dor intensa, em relação à dor ausente/leve/moderada, foi mais frequentemente referida pelos pacientes que tinham sintomas ansiosos, depressivos e entre os que tinham ao mesmo tempo os sintomas referidos $(\mathrm{p} \leq 0,05)$ (Tabela 3$)$.

A análise por regressão linear simples, tendo a pontuação de dor como variável dependente, indicou que a intensidade da dor é alterada em 0,25 para cada ponto de ansiedade aumentado ou diminuído e em 0,27 para cada ponto de depressão nas mesmas condições. O coeficiente de determinação $\left(r^{2}=0,19\right)$ foi semelhante tanto para a ansiedade como para a depressão $(\mathrm{p} \leq$ $0,05)$. O impacto da dor sobre a presença de sintomas ansiosos (Figura 1) e depressivos (Figura 2) pode ser demonstrado pela pontuação média crescente de cada uma, observada nas diferentes categorias de intensidade da dor. A curva é ascendente para as duas condições $(\mathrm{p} \leq 0,05)$.

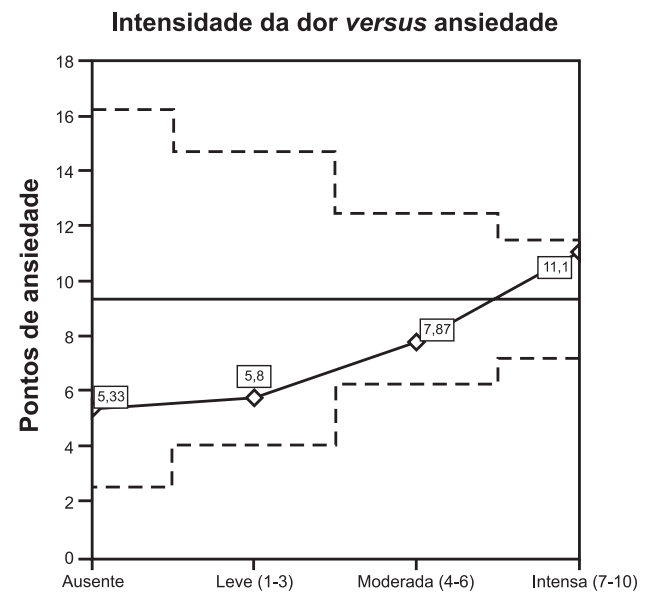

Figura 1 - Distribuição média de pontos de ansiedade entre as categorias de intensidade da dor $(p \leq 0,05)$

\section{Discussão}

Os pacientes portadores de neuropatia periférica podem sofrer tanto pela incapacitação funcional quanto pela dor trazida pela doença. A presença de ansiedade e/ou depressão pode modificar negativamente a história natural dessa enfermidade. No presente estudo, estimou-se uma frequência de $68,5 \%$ de sintomas de ansiedade e $51,9 \%$ de sintomas de depressão entre os pacientes portadores de neuropatia periférica, achado que é superior aos encontrados em estudos com pacientes clínicos de forma geral ${ }^{6,13,21,22}$. Comparados com estudos que abordaram neuropatias específicas, tais valores permanecem maiores ${ }^{12,23}$. Um estudo avaliando pacientes portadores de neuropatia diabética, com um desenho semelhante ao deste trabalho, estimou prevalências muito semelhantes: $60,4 \%$ para ansiedade e $50,6 \%$ para depressão ${ }^{10}$. Deve-se considerar que a mostra investigada no atual estudo envolveu uma população de baixa renda, a maioria com renda mensal familiar de, no máximo, 3 salários mínimos, afastada do emprego e apresentando comorbidades, muitas vezes, significativas.

Quase a metade dos pacientes do estudo apresenta tanto sintomas depressivos quanto ansiosos. A associação entre ansiedade e depressão é bastante conhecida e comum, sobretudo nos pacientes depressivos que procuram serviços médicos. Saber reconhecer um transtorno de ansiedade em um paciente depressivo é de extrema importância para uma conduta terapêutica mais eficaz ${ }^{24}$. A mesma cautela quanto ao diagnóstico e tratamento deve ser implementada para os transtornos bipolares, que podem se associar a transtornos de ansiedade em muitos casos. Preconiza-se, nesses casos, um controle prévio com estabilizadores de humor antes do uso de antidepressivos

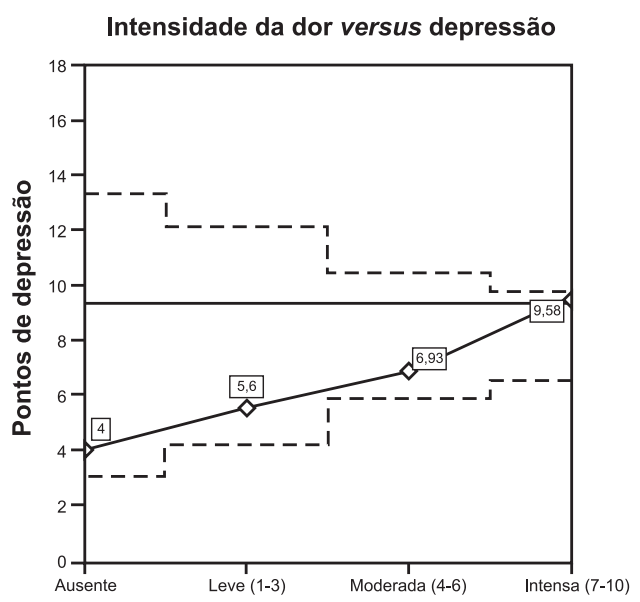

Figura 2 - Distribuição média de pontos de depressão entre as categorias de intensidade da dor $(\mathrm{p} \leq 0,05)$ 
para tratar os sintomas de ansiedade para que se evite o quadro maníaco induzido pelos últimos ${ }^{25}$.

História familiar de doença psiquiátrica associou-se positivamente com a presença de sintomas ansiosos e depressivos. Tem-se estudado fatores genéticos que possam explicar a sucessão de casos de ansiedade e/ou depressão em famílias, assim como a presença de sintomas ansiosos associados aos depressivos em um mesmo paciente. Estudos em gêmeos sugerem uma possível etiologia genética para a associação. Deve-se lembrar de que fatores ambientais específicos podem favorecer a eclosão de ambas as sintomatologias em determinados contextos familiares ${ }^{26}$.

A queixa de cefaleia como comorbidade se associou positivamente com a presença de sintomas depressivos e ansiosos - achado que coincide com outros estudos ${ }^{18,27}$. Propõe-se que a proximidade entre a cefaleia (especificamente a do tipo tensional) e a depressão se origine de uma disfunção das vias serotoninérgicas, presente nas duas condições. Deve-se considerar ainda o fator ansiogênico próprio do receio, referido por muitos pacientes que apresentam cefaleia importante, de que possam estar portando uma patologia mais grave (tumor cerebral, doença cerebrovascular) ${ }^{27}$.

A relação entre a ansiedade e a depressão com a dor neuropática já é conhecida, sendo mais frequente na literatura a avaliação do quadro depressivo ${ }^{10-12,28-30}$. Neste estudo, observou-se uma correlação positiva entre intensidade de dor e sintomas de ansiedade e depressão, sugerindo que, em pacientes com dores mais intensas, a intervenção terapêutica para os sintomas de ansiedade e depressão pode ser ainda mais necessária. Sabe-se que tanto a ansiedade quanto a depressão podem atuar em nível central como facilitadores das aferências dolorosas, participando da patogênese da dor ${ }^{24}$. O estudo da fisiopatologia depressiva associada à dor já determina uma aproximação estreita entre a regulação do humor e a percepção dolorosa. Atribui-se um papel importante aos distúrbios da neurotransmissão serotoninérgica e noradrenérgica, assim como a neuropeptídeos como opioides e substância P. Estruturas cerebrais, como a amígdala, o córtex cingulado anterior e o córtex pré-frontal, têm significado nesse processo. A ação dos antidepressivos duais em tais quadros deriva dessa patogênese $\mathrm{e}^{31}$.

A presença de neuropatias pós-traumáticas também se associou positivamente com a presença de sintomas de ansiedade e depressão. A perda ou deficiência súbita da função de um membro decorrente do trauma pode estar implicada nesse fenômeno, o que é agravado se o paciente é jovem, repercutindo em perda da atividade laboral. A presença de ansiedade e depressão ou a intensidade da dor não se correlacionaram significativamente com idade, sexo, estado civil, grau de escolaridade, religião, renda mensal familiar, duração da doença e presença de HAS, alcoolismo ou tabagismo no presente estudo.

Algumas limitações deste trabalho devem ser ressaltadas. $\mathrm{O}$ desenho transversal não permite definir relações causais entre a presença de dor e sintomas de ansiedade e depressão. O tamanho pequeno da amostra impossibilitou a análise multivariada, de forma que não foi possível avaliar o comportamento das variáveis do estudo entre as diferentes neuropatias periféricas. Uma avaliação mais pormenorizada do quadro psiquiátrico e de outras variáveis clínicas (como o índice de qualidade de vida e do sono) não pôde ser feita nesse estudo. A leitura oral do questionário pelo pesquisador pode ter gerado um viés de resposta, uma vez que se perde a espontaneidade e a autenticidade, mais observadas quando o indivíduo responde isoladamente.

A despeito das limitações, os resultados sugerem que as neuropatias periféricas estão associadas a uma elevada prevalência de sintomas ansiosos e depressivos e que a intensidade da dor neuropática pode influenciar na intensidade dos sintomas psiquiátricos. A abordagem desses pacientes deve ser diferenciada, atentando-se para dados clínicos específicos, como presença de cefaleia, antecedentes familiares de doença psiquiátrica, envolvimento ou não de trauma e intensidade da dor. A psico e farmacoterapia precisam ser melhor estudadas e indicadas para tais pacientes. Há poucos trabalhos envolvendo a psicoterapia no tratamento dos transtornos da ansiedade e depressão em pacientes com dores crônicas. Médicos generalistas e especialistas precisam saber reconhecer tais transtornos e definir um tratamento específico ou encaminhar o paciente para um serviço especializado. Espera-se que os dados obtidos por novos estudos possam ajudar a estabelecer rotinas para o exame e tratamento adequados do paciente portador de neuropatia periférica, reduzindo o risco de complicações e aumentando a eficácia terapêutica.

\section{Referências}

1. Lipowski ZJ. Consultation-liaison psychiatry: the first half century. Gen Hosp Psychiatry. 1986;8(5):305-15.

2. Azevedo AC. O estresse psicológico e o coração. Arq Bras Cardiol. 1993;60(4):211-4.

3. Popkin MK, Callies AL, Colon EA, Stiebel V. Adjustment disorders in medically ill inpatients referred for consultation in a university hospital. Psychosomatics. 1990;31(4):410-4.

4. Robinson RG, Starr LB, Kubos KL, Price TR. A two-year longitudinal study of post-stroke mood disorders: findings during the initial evaluation. Stroke. 1983;14(5):736-41.

5. Vega Alonso AT, Larrañaga Padilla M, Zurriaga Lloréis O, Gil Costa M, Urtiaga M, Calabuig Pérez J. Trastornos de ansiedad: variabilidad y condicionantes de la actitud terapéutica de los médicos de atención primaria. Aten Primaria. 1999;24(10):569-78.

6. Brasil MA, Furlanetto LM. A atual nosologia psiquiátrica e a sua adequação ao hospital geral. Cadernos IPUB. 1997;6:59-70.

7. Furlanetto LM. Diagnosticando depressão em pacientes internados em enfermarias de clínica médica. J Bras Psiquiatr. 1996;45(6):363-70.

8. Furlanetto LM, von Ammon Cavanaugh S, Bueno JR, Creech S, Powell LH. Association between depressive symptoms and mortality in medical inpatients. Psychosomatics. 2000;41(5):426-32.

9. Furlanetto LM, Cigognini MA. Diagnóstico e tratamento dos transtornos depressivos em hospital geral. Rev Bras Psiquiatr. 2006;28(2):97-103.

10. Gore M, Brandenburg NA, Dukes E, Hoffman DL, Tai KS, Stacey B. Pain severity in diabetic peripheral neuropathy is associated with patient functioning, symptom levels of anxiety and depression, and sleep. J Pain Symptom Manage. 2005;30(4):374-85.

11. Vileikyte L, Leventhal H, Gonzalez JS, Peyrot M, Rubin RR, Ulbecht JS, et al. Diabetic peripheral neuropathy and depressive symptoms: the association revisited. Diabetes Care. 2005;28(10):2378-83.

12. Williams LS, Jones WJ, Shen J, Robinson RL, Weinberger M, Kroenke K. Prevalence and impact of depression and pain in neurology outpatients. J Neurol Neurosurg Psychiatry. 2003;74(11):1587-9. 
13. McWilliams LA, Cox BJ, Enns MW. Mood and anxiety disorders associated with chronic pain: an examination in a nationally representative sample. Pain. 2003;106(1-2):127-33.

14. Bair MJ, Robinson RL, Katon W, Kroencke K. Depression and pain comorbidity: a literature review. Arch Intern Med. 2003;163(20):2433-45.

15. Gureje O, Von Korff M, Kola L, Demyttenaere K, He Y, Posada-Villa J, et al The relation between multiple pains and mental disorders: results from the World Mental Health Surveys. Pain. 2008;135(1-2):82-91.

16. Von Korff M, Crane P, Lane M, Miglioretti DL, Simon G, Saunders K, et al. Chronic spinal pain and physical-mental comorbidity in the United States: results from the national comorbidity survey replication. Pain. 2005;113(3):331-9.

17. McWilliams LA, Goodwin RD, Cox BJ. Depression and anxiety associated with three pain conditions: results from a nationally representative sample. Pain. 2004;111(1-2):77-83.

18. Snaith RP, Zigmond AS. The hospital anxiety and depression scale. Br Med J (Clin Res Ed). 1986;292(6516):344.

19. Botega NJ, Pondé MP, Medeiros P, Lima MG, Guerreiro CAM. Validação da escala hospitalar de ansiedade e depressão (HAD) em pacientes epilépticos ambulatoriais. J Bras Psiquiatr. 1998;47(6):285-9.

20. Tölle T, Xu X, Sadosky AB. Painful diabetic neuropathy: a cross-sectional survey of health state impairment and treatment patterns. J Diabetes Complications. 2006;20(1):26-33.

21. Tanajura D, Santos-Jesus R, Tavares-Neto J, Oliveira IR. Prevalence of depression in different groups of inpatients at the University Hospital of Bahia, Brazil. Rev Bras Psiquiatr. 2002;24(4):182-5.
22. Botega NJ, Bio MR, Zomignani MA, Garcia Jr. C, Pereira WAB. Transtornos de humor em enfermaria de clínica médica e validação de escala de medida (HAD) de ansiedade e depressão. Rev Saude Publica. 1995;29(5):355-63.

23. Pfeiffer G, Wicklein EM, Ratusinski T, Schmitt L, Kunze K. Disability and quality of life in Charcot-Marie-Tooth disease type 1. J Neurol Neurosurg Psychiatry. 2001;70(4):548-50.

24. Zimmerman M, McDermut W, Mattia JI. Frequency of anxiety disorders in psychiatric outpatients with major depressive disorder. Am J Psychiatry. 2000;157(8):1337-40.

25. Freeman MP, Freeman SA, McElroy SL. The comorbidity of bipolar and anxiety disorders: prevalence, psychobiology and treatment issues. J Affect Disord. 2002;68(1):1-23.

26. Hettema JM. What is the genetic relationship between anxiety and depression? Am J Med Genet C Semin Med Genet. 2008;148(2):140-6.

27. Matta APC, Filho PFM. Sintomas depressivos e ansiedade em pacientes com cefaléia do tipo tensional crônica e episódica. Arq Neuropsiquiatr. 2003;61(4):991-4.

28. Berger A, Dukes EM, Oster G. Clinical characteristics and economic costs of patients with painful neuropathic disorders. J Pain. 2004;5(3):143-9.

29. Fishbain DA, Cutler R, Rosomoff HL, Rosomoff RS. Chronic pain-associated depression: antecedent or consequence of chronic pain? A review. Clin J Pain. 1997;13(2):116-37.

30. Fishbain DA, Cutler BR, Rosomoff HL, Rosomoff RS. Comorbidity between psychiatric disorders and chronic pain. Curr Review Pain. 1998;2(1):1-10.

31. Jaracz J, Rybakowski J. [Depression and pain: novel clinical, neurobiological and psychopharmacological data]. Psychiatr Pol. 2005;39(5):937-50. 